\title{
Vulnerability and Resilience Research: A Critical Perspective
}

\author{
Hannes Taubenböck • Christian Geiß
}

Published online: 29 March 2014

(c) The Author(s) 2014. This article is published with open access at Springerlink.com

\begin{abstract}
"Vulnerability" and "resilience" are terms of such broad conceptual meaning as to be almost useless for careful scientific communication, except as rhetorical indicators of areas of greatest concern. This is a reflection of the complexity of their meaning, an uncoordinated search among different fields for a common understanding, and maybe the difficulty of systematizing these complicated issues among the various involved parties.

In a critical commentary about the field of vulnerability and resilience research one could expect a list of more or less obvious and not so obvious issues observed over the years within and beyond this specific research landscape: the lack of transparent contributions from the different disciplines involved, the lack of a common taxonomy and nomenclature, the bewildering amount of different epistemological frameworks, the problem of rather abstract conceptual components versus measurable components, the unwillingness to engage in multi- and transdisciplinary understanding and collaboration, the often unclear responsibilities of the stakeholders involved, the neglecting of target-oriented research, and so on; however, it is not our intention to discuss or enlarge this list of examples.

With the designation of the 1990s as the International Decade for Natural Disaster Reduction, the United Nations General Assembly recognized the urgent goal of attenuating the impact of natural disasters. Since then, an almost unmanageable number of research projects and initiatives have been carried out. Current and future projects will enlarge this body of vulnerability and resilience research.
\end{abstract}

H. Taubenböck $(\bowtie) \cdot$ C. Geiß

German Remote Sensing Data Center, German Aerospace

Center, Oberpfaffenhofen, 82234 Wessling, Germany

e-mail: hannes.taubenboeck@dlr.de
Thus, it is an almost impossible task to gain a comprehensive overview of these projects and initiatives (respectively their developed and applied frameworks, concepts, approaches, data, methods, results, and applications); of all the institutions, stakeholders, funding agencies, and people involved; and of the different levels of political responsibility and participation.

A first and crucial step forward would be to gather information on all of these projects and initiatives in a systematic and structured way. This compendium of information would gain value if crucial conclusions for future progress were drawn-not only by individual researchers or disciplines, but especially by and beyond the research and stakeholder community in general. We are unable at this stage to list the relevant and crucial conclusions, but we would like to suggest ideas on how to get there:

\section{Science on a Meta-Level: Research About Research is Necessary and Much-Needed!}

The multi- and transdisciplinary community analyzes specific questions about, for example, physical, demographic, social, economic, environmental, institutional, or political vulnerability and resilience with respect to different hazards such as geophysical, meteorological, hydrological, or climatological events-as this special issue also indicates. No doubt, every individual research question and every individual approach to solve the respective question is of crucial importance. But are we losing integration of the bits and pieces of research into the necessary holistic and general overview along the way?

Benchmarking of scientific contributions is largely absent. Projects are too often realized on isolated questions 
failing to integrate them according to higher-ranking goals or frameworks. Too often projects are concurrently running on the same topic. Identification of research gaps and promising solutions, as well as the documentation of tested, but failed approaches too often does not occur. There is no culture of also publishing blind alleys.

However, research about research is crucial to assemble the status quo. Systematization of this information with the aim of identifying future priorities for a multi-disciplinary research landscape is essential. In addition to scientific advice, user requirements, technical issues, people's needs, and political ideas and directions, this information can help us draw clearer and more specific conclusions for welldirected, transparent goals.

This is an ambitious but necessary goal. Manifold different disciplines and stakeholders are involved, with different levels of abstraction and perspectives and different schools of thinking, concepts, methods, and data. This makes the task of reviewing and benchmarking of and concluding from scientific contributions highly complex and bulky.

With a smaller scope and on a lower level of abstraction we have dared to suggest conclusions based on an extensive review of the status quo within a section of our own research field. Coming from the field of remote sensing, our work has focused on the capabilities of this single discipline, and, if appropriate, its capabilities of multidisciplinary value-adding to contribute to earthquake risk analysis (Geiß and Taubenböck 2013). We aimed to give a comprehensive thematic and quantitative review on the current scientific status quo, listed and reviewed relevant projects and initiatives, and interviewed stakeholders involved. Working on this review to draw conclusions not only for scientific remote sensing issues, but also on technical, multi- and transdisciplinary as well as political issues, the complexity of the suggestion for value-adding presented above became clear. Although our area of research about research was limited to only remote sensing and earthquake risk issues, we found and reviewed more than 300 peer-reviewed articles and included more than 40 initiatives and projects, which-in our opinionhave contributed significantly to the field of remote sensing and earthquake risk.

Even at this lower level of abstraction, this example proves that putting together a comprehensive or nearly complete picture is a very complex task, and the conclusion for suggesting a road map is at risk of being incomplete, unbalanced, or biased. The conclusions may not conform with many schools of thinking and involved parties. However, we think, this risk has to be taken by the science community for the science community as well as the stakeholders, with a plea for an open and honest dialogue aimed at well-directed research! Now!

Keeping the consciously chosen polemic way of writing this comment, we argue for a stop of professionalized but uninspired producing of frameworks, concepts, approaches, and applications that sometimes lack any kind of exigency. From our point of view the most urgent action would be to step back and critically ask: what for?

Let's assume the problem may not be the identification of clear goals and promising research directions; the problem may rather be the connection between the identification of these clear goals, and their transformation into reality, and its needed acceptance. Let's start now!

Open Access This article is distributed under the terms of the Creative Commons Attribution License which permits any use, distribution, and reproduction in any medium, provided the original author(s) and the source are credited.

\section{Reference}

Geiß, C., and H. Taubenböck. 2013. Remote sensing contributing to assess earthquake risk: From a literature review towards a road map. Natural Hazards 68(1): 7-48. 